Supporting Information

\title{
Metal Microfibers Delivered Eddy Current Heating for Efficient Synthesis and Regeneration of Metal-Organic Framework Monoliths
}

Qiangqiang Li, Qiannan Wu, Yingle Tao, and Haiqing $L i *$

State Key Laboratory of Materials-Oriented Chemical Engineering, College of

Chemical Engineering, Nanjing Tech University, Nanjing 211816, China

E-mail: haiqing.li@njtech.edu.cn 

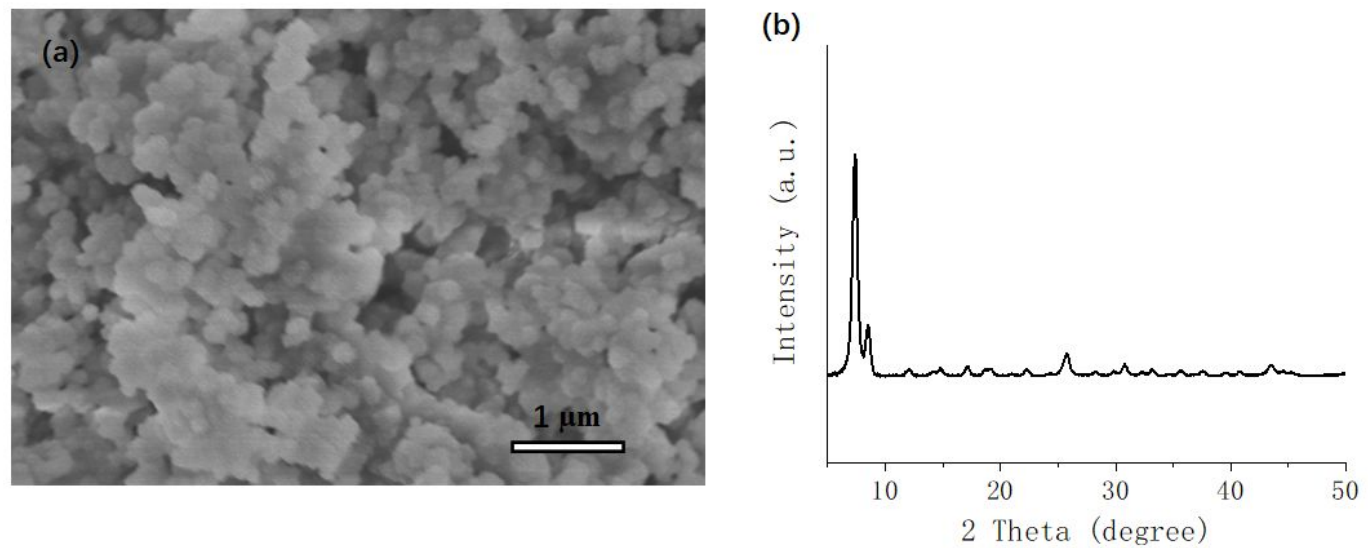

Figure S1. SEM image (a) and XRD pattern (b) of bare UiO-66- $\mathrm{NH}_{2}$ synthesized through the traditional solvothermal reaction. 


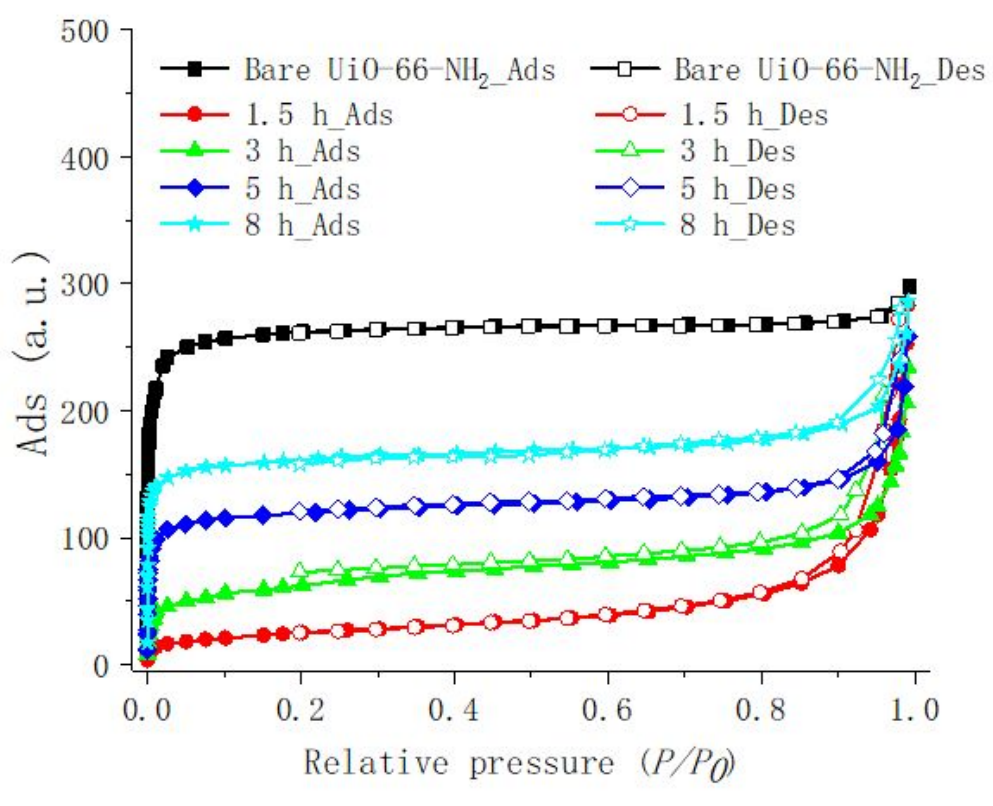

Figure S2. $\mathrm{N}_{2}$ adsorption isotherms of bare UiO-66- $\mathrm{NH}_{2}$ obtained with traditional solvothermal reaction and SSF/UiO-66- $\mathrm{NH}_{2}$ composites synthesized by LIFS for different reaction time. LIFS reaction was carried out with $0.66 \mathrm{mT}$ of magnetic field applied. 


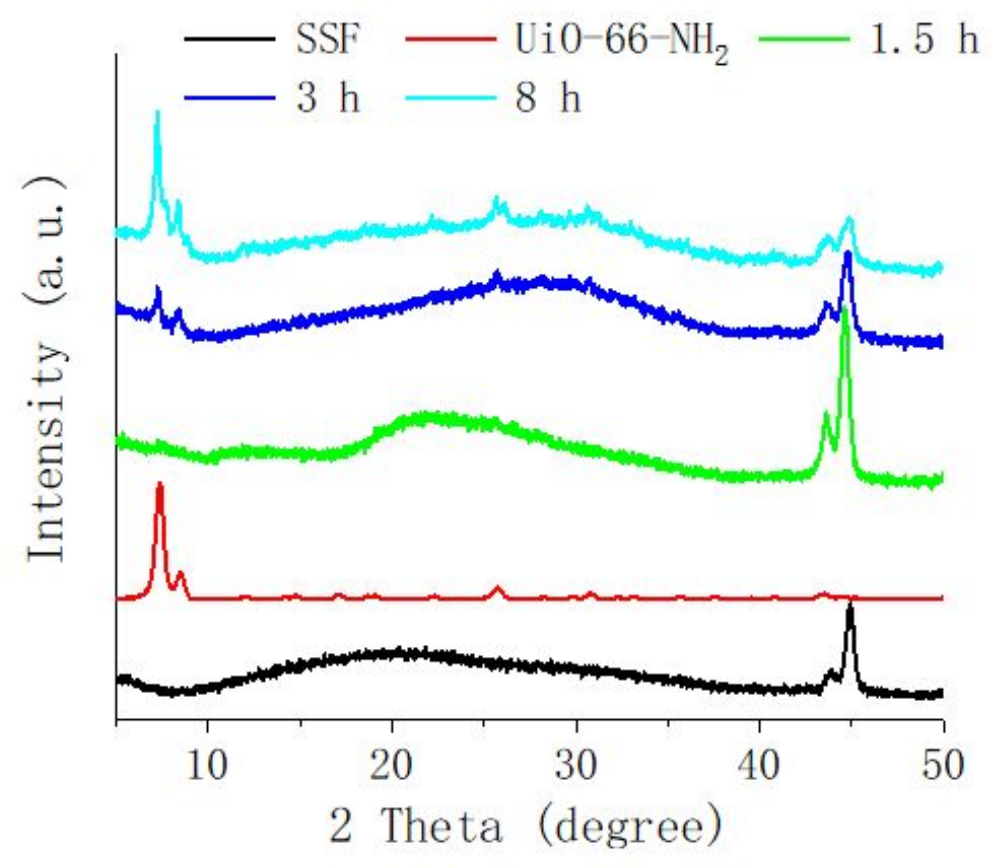

Figure S3. XRD patterns of SSFs, bare UiO-66- $\mathrm{NH}_{2}$, and SSF/UiO-66- $\mathrm{NH}_{2}$ composites synthesized through the traditional solvothermal reaction at $100{ }^{\circ} \mathrm{C}$. 
(a)
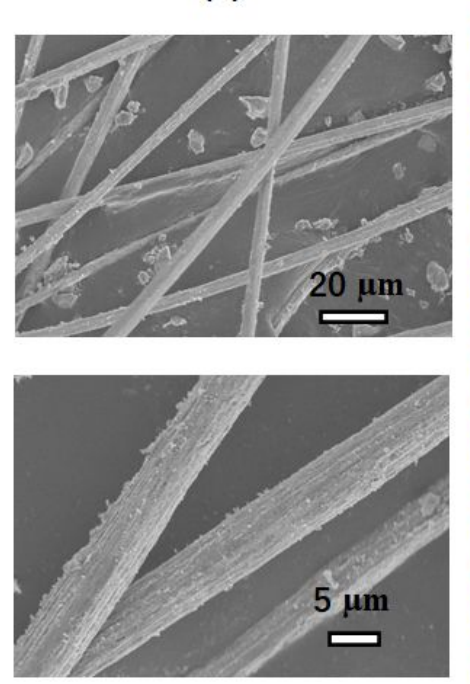

$3 \mathrm{~h}$ (b)

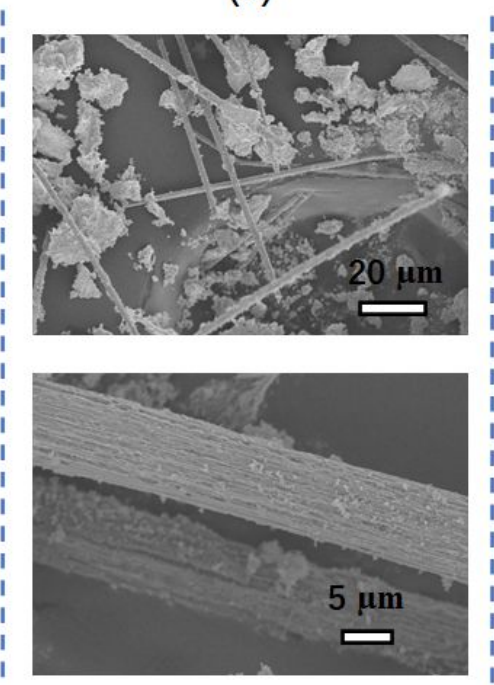

$5 \mathrm{~h}$
(C)
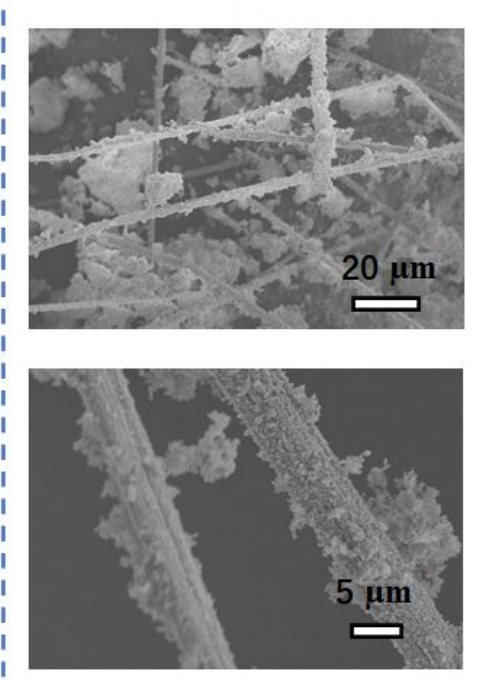

$8 \mathrm{~h}$

Figure S4. SEM images of SSF/UiO-66- $\mathrm{NH}_{2}$ composites synthesized through the traditional solvothermal reaction at $100^{\circ} \mathrm{C}$. 

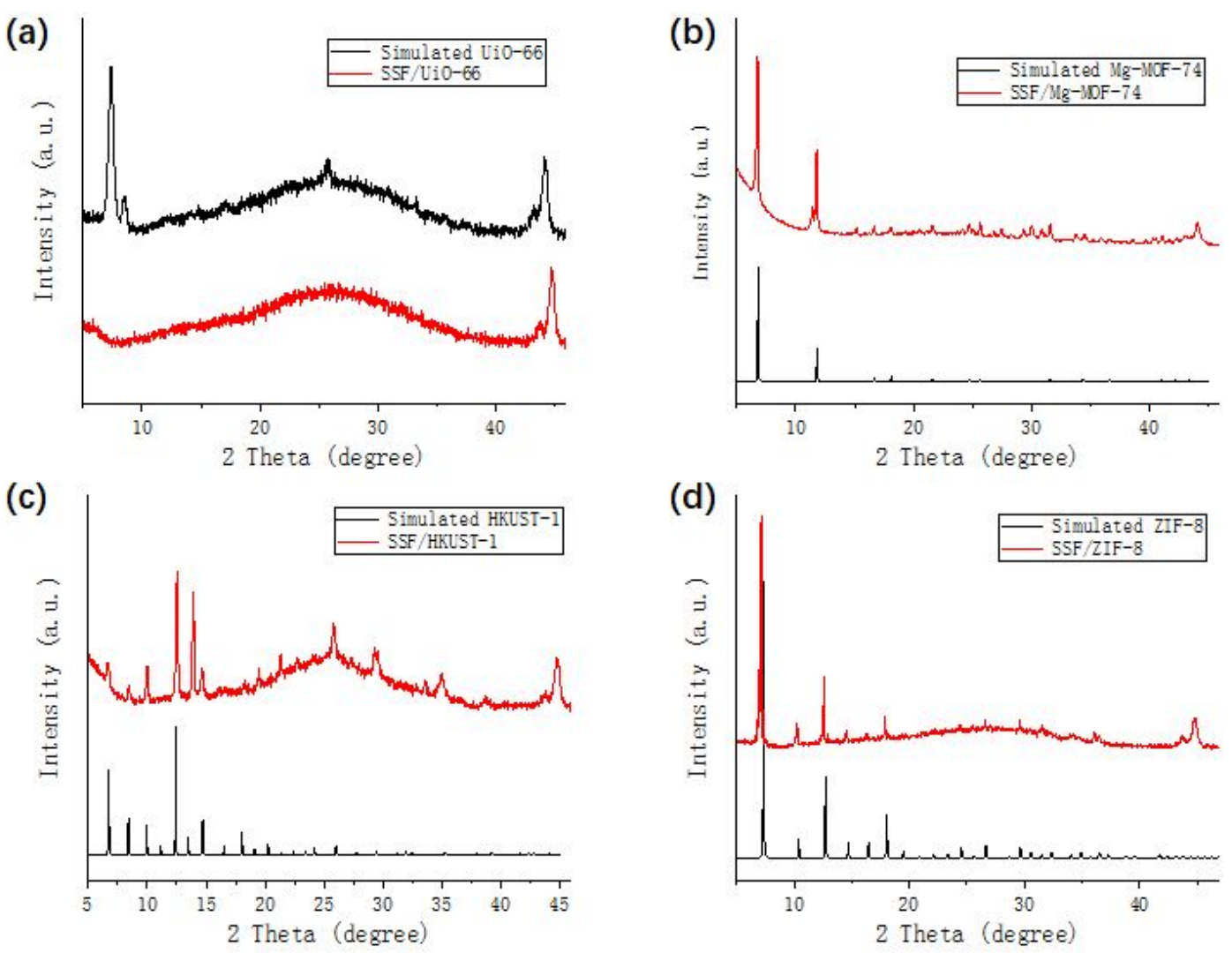

Figure S5. XRD patterns of varied SSF/MOF composites synthesized by LIFS reactions with $0.66 \mathrm{mT}$ of magnetic field applied. 


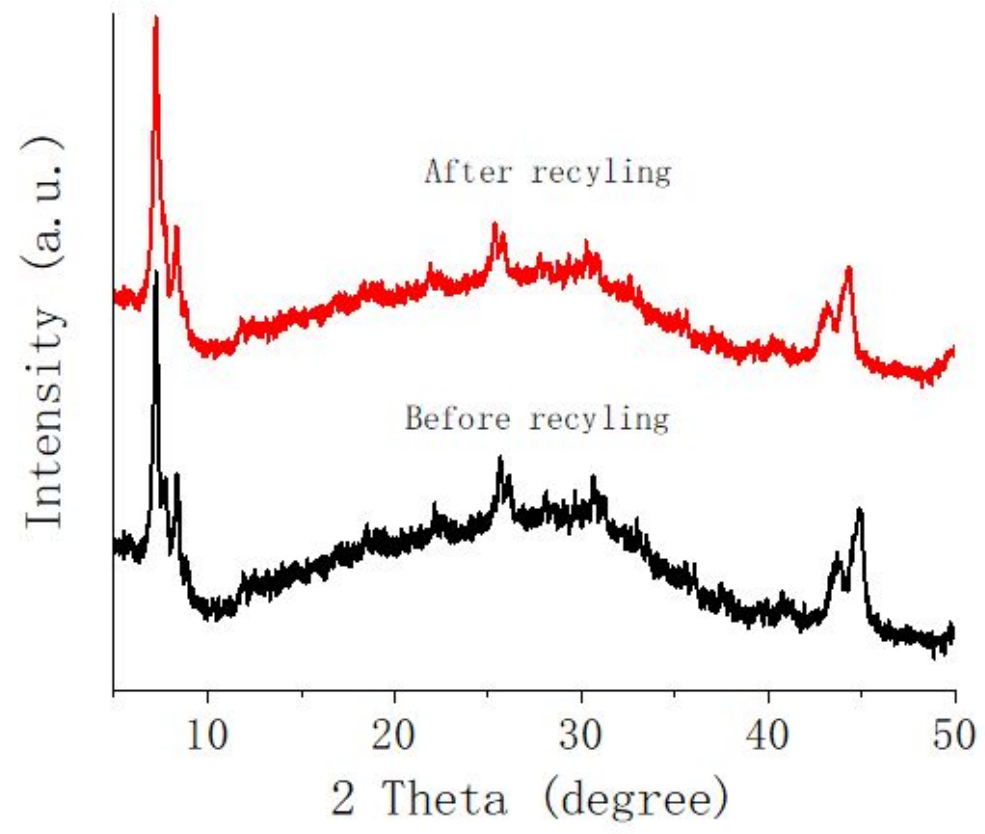

Figure S6. XRD patterns of SSF/UiO-66- $\mathrm{NH}_{2}$ obtained by $8 \mathrm{~h}$ of LIFS reaction before and after 5 cycles of reuse. 
(a)
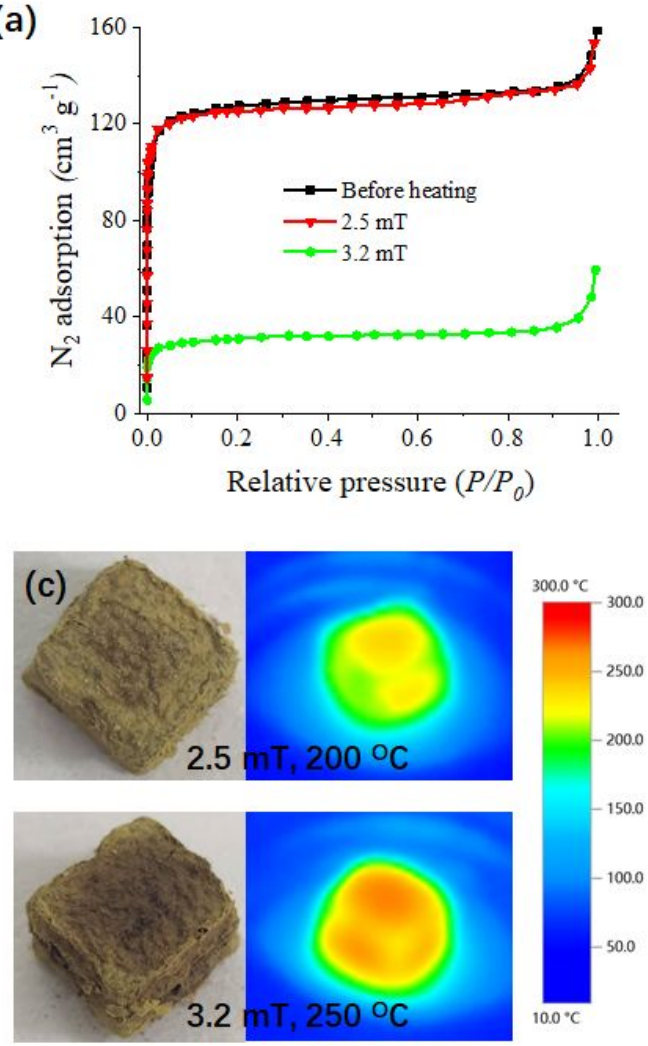

(b)
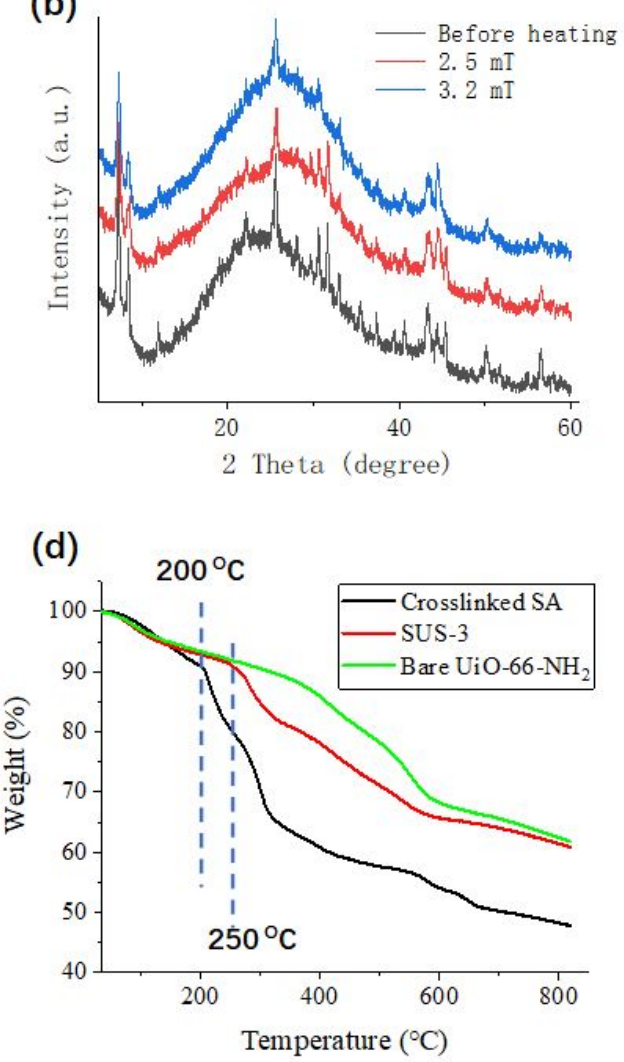

Figure S7. $\mathrm{N}_{2}$ adsorption isotherms at $77 \mathrm{~K}$ (a) and XRD patterns (b) of SUS-3 before and after applying 2.5 and $3.2 \mathrm{mT}$ of magnetic field. Digital image and the corresponding IR image of SUS-3 after expositing to 2.5 and $3.2 \mathrm{mT}$ of magnetic field (c), and TGA profiles of bare UiO-66-NH2, crosslinked SA, and SUS-3 (d). Upon application of a $2.5 \mathrm{mT}$ of magnetic field, SUS-3 was heated up to $200^{\circ} \mathrm{C}$. No obvious changes in its surface area (around $440 \mathrm{~m}^{2} \mathrm{~g}^{-1}$ ) and XRD patterns were observed. In contrast, application of a stronger magnetic field $(3.2 \mathrm{mT})$ to SUS-3 resulted in its temperature increased up to $250^{\circ} \mathrm{C}$. Under such heating condition, BET surface area of SUS-3 dramatically dropped down to $96 \mathrm{~m}^{2} \mathrm{~g}^{-1}$, while no obvious alternations in its XRD patterns were observed. Due to the lower thermal stability of crosslinked SA component (red line in (d)), the application of $3.2 \mathrm{mT}$ of magnetic field to SUS-3 would decompose its crosslinked SA component, while the crystal structure of its MOF 
component still remained largely unaltered due to the better thermal stability of MOF (red line in (d)). Therefore, such a significant drop in the surface area of SUS-3 might be caused by the blockage of MOF channels rendered by the decomposed SA fragments.

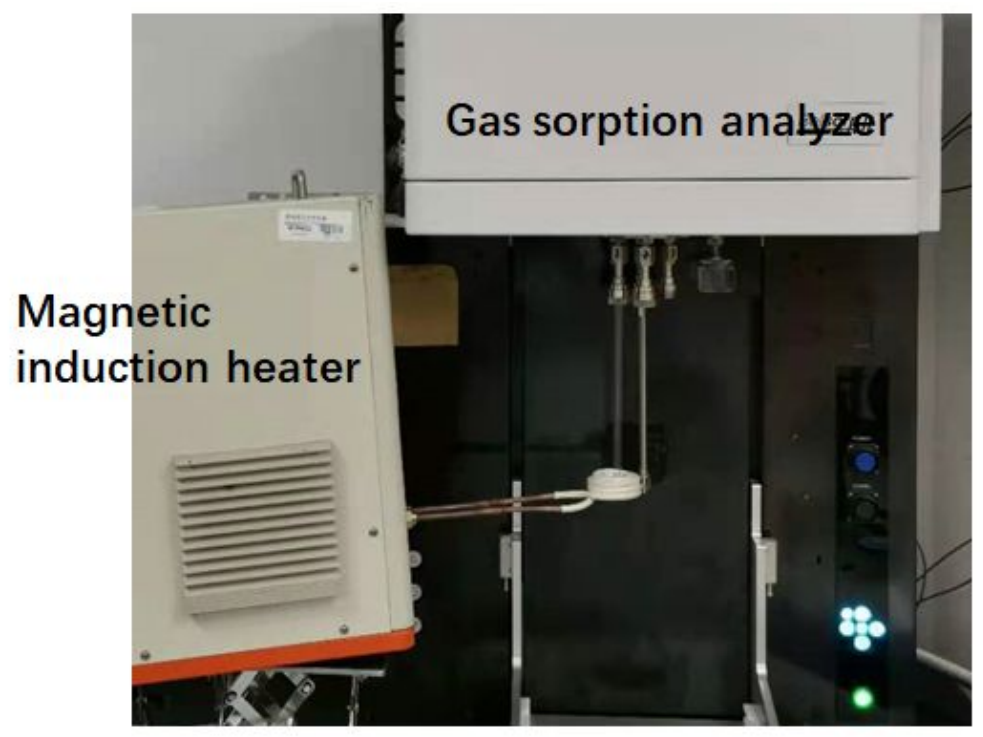

Figure S8. Digital image of gas sorption analyzer equipped with a magnetic induction heater for $\mathrm{CO}_{2}$ adsorption isotherms measurements with/without a magnetic field applied. 




Figure S9. $\mathrm{CO}_{2}$ adsorption of bare UiO-66- $\mathrm{NH}_{2}$ and SUS-3 with and without a 2.1 mT of magnetic field applied. The measurements were carried out at $298 \mathrm{~K}$. 